\title{
Whole class vs. small group settings for using animations in physics: Case study comparisons
}

\author{
A. Lynn Stephens and John J. Clement \\ College of Education, University of Massachusetts-Amherst \\ Hills North, Thatcher Road, Amherst, MA, 01003
}

\begin{abstract}
Comparative case study analyses are used to investigate a physics lesson sequence in which students used a simple simulation and a set of animations with playback controls to explore aspects of projectile motion. The sequence was conducted within naturalistic high school settings ( 2 schools) in 11 physics class sections $(n=212)$ where roughly half the sections encountered the animations in a whole class discussion format and matched sections used them in a small group format in which students controlled playback. Earlier unexpected pre-post results indicated no advantage for the hands-on condition. Present analyses using classroom videotapes and student written work also do not show any overall advantage for the small group students for the factors examined. Notably, more than twice as much teacher and studentgenerated support for recognizing visual features was identified in whole class discussion, and in small groups these episodes appeared to cluster around teacher visits to the groups.
\end{abstract}

PACS: $01.40 . \mathrm{Fk}, 01.50 . \mathrm{ht}, 01.40 . \mathrm{gb}, 01.40 . \mathrm{ek}$

\section{INTRODUCTION}

Studies have suggested that students benefit from control of the pace of animations; the speed of a presentation needs to match the speed of comprehension of a topic (e.g., Mayer and Chandler [1]). Small group work has been prized for allowing such control as well as for providing students opportunities to interact with others, to create metaphors that other students can readily understand, and to enter engaged exploration of the concepts of a lesson. On the other hand, we have observed teachers scaffold interesting discussions about animations in both small group and whole class contexts. In a related study [2], we looked at small group and whole class response to a sophisticated, highly interactive physics simulation (where students could vary multiple parameters); here we look at response to animations (interaction restricted to playback controls), among a larger group of high school physics students and teachers. For matched classes (same teacher, similar course preparation and ability of students as determined by teacher and research team) taught in either a whole class discussion or a small group mode:

- To what extent did students and teachers engage in discussion about certain crucial concepts addressed in the animations?

- To what extent did teachers and students attempt to respond to conceptual difficulties and misconceptions?

- To what extent did teachers and students support the recognition and/or interpretation of key visual features and visual relationships in the animations?

- Did students recognize and utilize the key visual features and relationships in their own thinking?

\section{BACKGROUND}

Although some researchers have investigated the effectiveness of animations when teachers provided the verbal information (e.g., Russell \& Kozma [3]), there do not appear to be many studies that address how best to provide instructional guidance for animations when used in a whole class setting. Principles suggested by theory and by laboratory work with animations (Lowe [4]; Mayer \& Moreno [5]) would appear to need further validation in science classroom contexts (Cook [6]), and may have to be modified to be usable by teachers employing available physics animations in whole class situations. Cognitive studies have indicated that comprehension of animations depends on students having control of the rate of flow of the information (Mayer and Chandler [1]; Schwan and Riempp [7]). Considering this, and the fact that the teachers in our study stated they prefer to allow students to work with physics animations in small groups and feel experienced teaching in that format, it might be expected that the small group format would work better for them than a whole class format. On the other hand, studies have reported a variety of issues concerning the effective use of small group discussions in science classes, such as that students can exhibit a low level of engagement with tasks (Bennett, Hogarth, Lubben, Campbell, and Robinson [8]).

\section{PARTICIPANTS AND SETTING}

Three teachers taught a lesson sequence on projectile motion to matched sets of class sections, $n=212$. Each set had one class taught in a small group format and at least one class taught in a whole class format. In the small group format, 2-4 students per computer explored the visuals 
using playback controls and engaged in discussion guided by the activity sheets while the teacher circulated among the groups. In the whole class format, the teacher used a single computer to project the visuals onto a screen in front of the class and facilitated a whole class discussion as students worked through two activity sheets. Materials were identical in the two conditions.

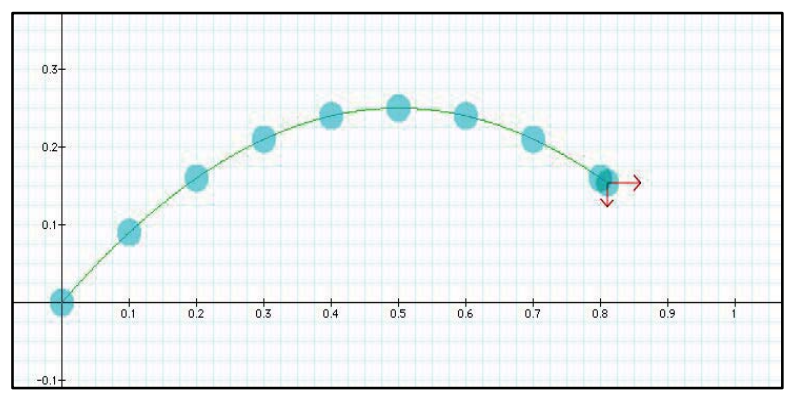

FIG 1. Screenshot of one of three projectile animations. Animated (red) arrows indicate components of motion.

TABLE 1. Matched class sections.

\begin{tabular}{lccc}
\hline Level & Teacher & Whole Class & Small Group \\
\hline Collge Prep & A & 1 & 1 \\
Honors (1) & A & 1 & 1 \\
Honors (2) & A & 1 & 1 \\
Honors (3) & B & 2 & 1 \\
Adv Placmt & C & 1 & 1 \\
\hline
\end{tabular}

College Preparatory (CP) was the least advanced class and Advanced Placement (AP) the most (Table 1). The lesson sequence varied from one to three days depending on teacher and physics level; time on task was controlled within each matched set by allowing each condition the same amount of time for each activity sheet and use of visual tools. A simple simulation, several animations, and manipulatives were used. All lessons were observed and videotaped. Pre/post tests and student worksheets were collected. 23 videotapes were collected and all were subjected to preliminary analysis. Similar issues were observed during the animation and simulation portions of the lesson but were particularly clearly exhibited during the animations portion. Therefore, in-depth analyses focused on the animations discussions and animations activity sheets.

\section{PRELIMINARY PRE/POST RESULTS}

Preliminary pre/post results from this and another lesson sequence (four comparisons; see Stephens [9]) provided further motivation for the study. To the surprise of the teachers, the results showed no significant advantage for the small group condition in any of the nine comparisons. If anything, there appeared to be a trend toward the whole class condition. These results are discussed in depth in Stephens [9] and motivated the present qualitative study.

\section{METHOD}

Videotape analysis was used to address the first three research questions. The video camera can be viewed as a proxy for an individual student; it took the viewpoint of a hypothetical student in that class and recorded what $\mathrm{s} / \mathrm{he}$ might have seen and heard. The discussion portions of the videotapes were coded using the codes summarized below. The results of this analysis can suggest hypotheses about factors at work in the two discussion formats.

Activity sheet analysis was used to address the fourth question to provide an estimate of how many students actually used the key visual features in their own thinking, as evidenced by their written and drawn answers to relevant activity sheet questions. This analysis includes all of the students in the classes, unlike videotape analysis.

A complete list of codes and examples are in Ref. [9].

\section{ANALYSIS AND RESULTS}

\section{A. Question one}

To what extent did students and teachers engage in discussion about certain crucial concepts addressed in the animations?

Codes: Student or teacher mentions concrete causal factor; i.e., student or teacher asks question about or mentions a concrete explanation as to why some aspect of the phenomena in the system under discussion is occurring.

An issue that emerged in preliminary classroom observations was that of student difficulty in identifying concrete causal factors for different aspects of projectile motion. We looked for any discussion of why projectiles behave as they do, explained in concrete terms, even if the suggested causal factor was not correct. Fig. 2 shows the results expressed as percentage of discussion time.

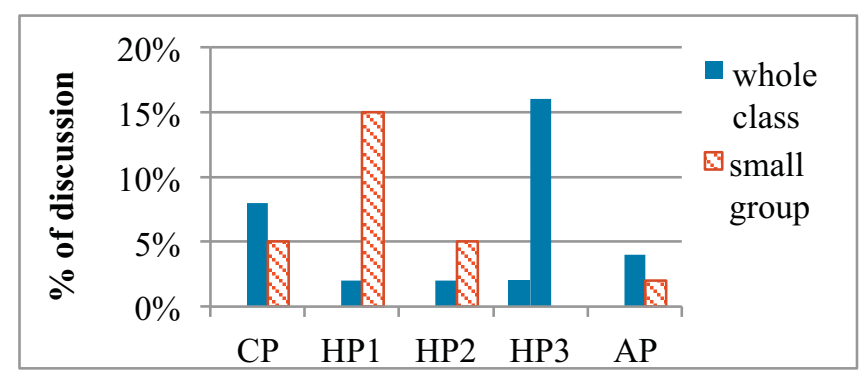

FIG 2. Discussion about crucial concepts as percentage of discussion time. HP3 had three matched discussions, but the small group discussion did not mention the concepts.

Coded portions ranged from $0 \%$ to $15 \%$ of discussion time in the small groups on camera and from $2 \%$ to $16 \%$ in the whole class discussions, not exceeding 3 minutes in any discussion. In 3 of 5 comparisons, the percentage of discussion time spent on the crucial concepts was greater in 
the whole class discussions than in the matched small group discussions. Percentages were used to adjust for the fact that small groups did not always use all the time available to them; the difference in length of time spent was more than this. This does not suggest an overall advantage on the measure for the small group students. One small group did not discuss the crucial concepts at all. We were surprised at how little the discussions addressed potential causal factors.

\section{B. Question two}

To what extent did teachers and students attempt to respond to conceptual difficulties and misconceptions?

Code: Response to conceptual difficulty. Classroom activity was considered a response if it bore some relationship to difficulty expressed by a student.

Code: Response to misconception. Classroom activity was considered a response to a misconception if it appeared to be an attempt to address a misconception.

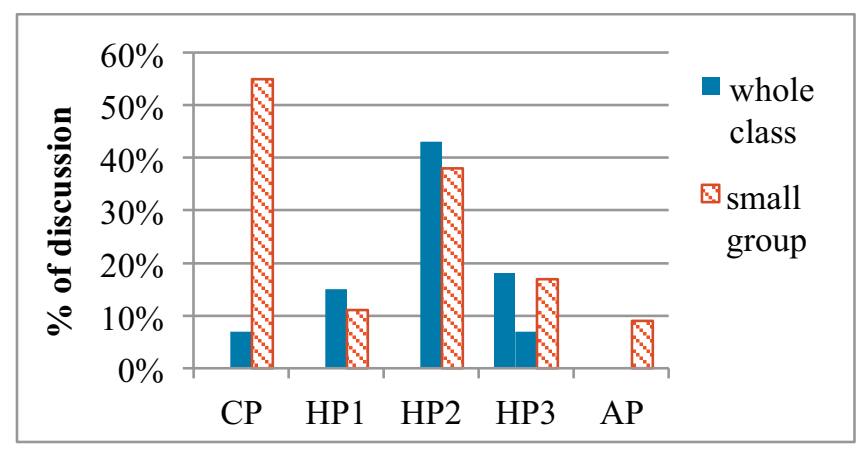

FIG 3. Response to conceptual difficulties as percentage of discussion time. The AP whole class discussion had none.

From $0 \%$ to $55 \%$ of discussion times were spent addressing student difficulties (Fig. 3), ranging from 0 to almost 16 minutes. In neither Advanced Placement class was there evidence of much conceptual difficulty; not surprisingly, there was little or no response time. The interesting result is for the lower level CP class where the small group analyzed spent over $50 \%$ of their time discussing conceptual difficulties: video analysis revealed that for this small group, the teacher was present almost the entire time difficulties were being addressed.

\section{Question three}

To what extent did teachers and students support the recognition and/or interpretation of key visual features and visual relationships in the animations?

Codes: Student or teacher supports identification of a key visual feature or relationship in an animation. By this, we mean support for recognition of its existence; e.g., using gestures to indicate a visual relationship to another student.

Codes: Student or teacher supports interpretation of a key visual feature or relationship in an animation. By "interpretation" of a key feature, we mean the interpretation of its meaning, the development of some degree of understanding (as opposed to attaining rote knowledge of the feature or the ability to recreate a visual aspect through mimicry); e.g., the recognition that the behavior of the animated (red) arrows in Fig. 1 could indicate the presence of acceleration.

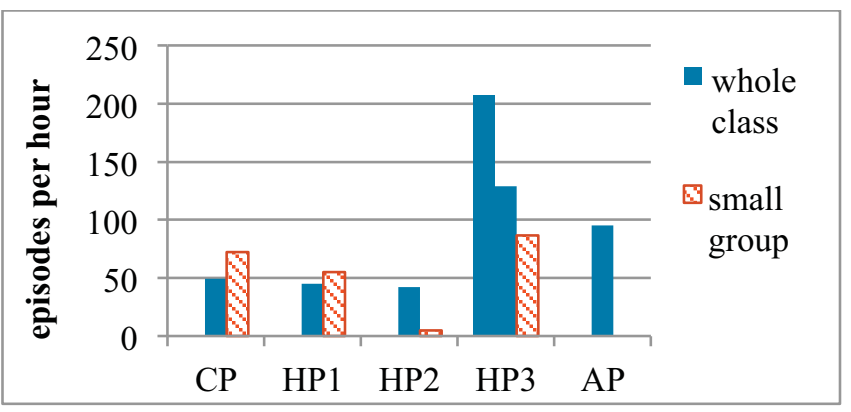

FIG 4. Support for key visual features as episodes per hour. The AP small group discussion had no support episodes.

This analysis identified 'episodes' (typically quite brief, lasting a few seconds; see Fig. 6). Results indicated a higher rate of visual support episodes in 3 out of 5 comparisons (Fig. 4). The total numbers of these episodes are shown in Table 2. Note that for HP3, both whole class discussions had far more episodes and greater frequency than the matched small group discussion analyzed. The small groups in HP2 and AP had one and zero support episodes, respectively, while the matched whole class discussions had 25 and 21. These results do not suggest an overall advantage on this measure for the small group students.

TABLE 2. Support for key visual features as total number of episodes per discussion.

\begin{tabular}{lccc}
\hline Level & Teacher & Whole Cl & Small G \\
\hline CP & A & 15 & 23 \\
HP1 & A & 12 & 11 \\
HP2 & A & 25 & 1 \\
HP3 & B & 54,40 & 25 \\
AP & C & 21 & 0 \\
\hline
\end{tabular}

\section{Question four}

Did students recognize and utilize the key visual features and relationships in their own thinking?

We analyzed the student drawing and writing on the activity sheets that accompanied the animations portion of the lesson. The key features and relationships identified in the three animations included such aspects as the (red) arrows shown in Figure 1 and how they changed with time.

Four questions on the activity sheet were selected for analysis for their potential to shed light on whether students actually understood what the features meant and whether they had grasped a central concept addressed by the 
features, that projectiles accelerate in the downward direction only. All activity sheets that included answers to these questions were coded, 212 sheets. All coding was done blind to whole class or small group condition.

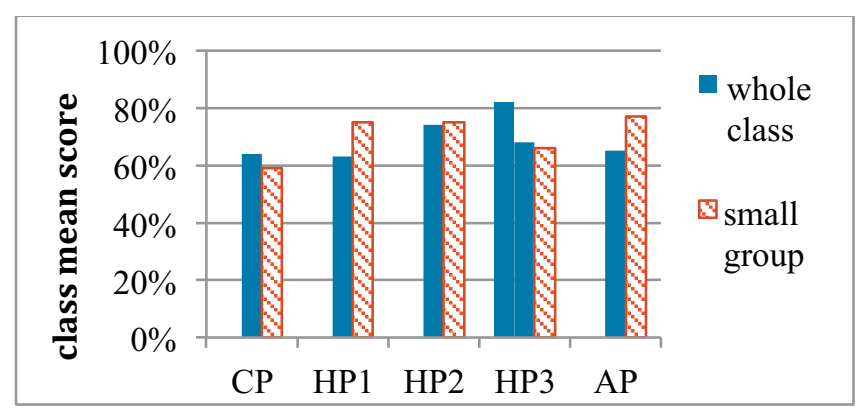

FIG 5. Class mean performance on four animation activity sheet questions as percentage of a perfect score.

Scores were very similar across all classes. Most students in both lesson formats exhibited considerable understanding about the meanings and implications of the visual features and visual relationships. There did not appear to be an advantage for students in the small group condition over those in the whole class condition (Fig. 5).

\section{DISCUSSION AND CONCLUSIONS}

Although video analysis revealed considerable variation among groups, there was no evidence for an overall advantage for the small groups in this study. These findings complement those of Stephens and Clement [2], which also showed, in a somewhat different context, no evidence for an advantage for hands-on work with a visual tool over utilizing the tool in a whole class discussion format. There, too, the whole class discussions had many more visual support episodes than the small groups analyzed. While the earlier study involved a sophisticated highly interactive computer simulation, the present study investigated what would happen if we used a simpler tool that included fewer features and fewer moving elements on screen. The present study also included a third teacher and larger $n$.

A striking observation was the clustering of support codes around teacher visits to the small groups. For example, the code map of the discussion from Teacher B's HP3 small group class shows that in an 18-minute

[1] R. E. Mayer and P. Chandler, J. Educ. Psychol. 93, 390 (2001).

[2] A. L. Stephens and J. J. Clement, Comput.Educ. 86, 137 (2015).

[3] J. Russell and R. Cosma, in Visualization in science education, edited by J. K. Gilbert (Springer, Dordrecht, 2005).

[4] R. K. Lowe, Learn. Instr. 13, 157 (2003).

[5] R. E. Mayer and R. Moreno, Educ. Psychol. 14, 87 discussion, almost all the analytical codes, including those for student-to-student support, occurred between minutes 9 - 13 when the teacher was present with the group (Fig. 6). This clustering was observed for both of the small group discussions we analyzed in which a teacher stopped by. (The other was Teacher A's CP small group.) On the other hand, such conceptual and perceptual support was generally observed throughout the whole class discussions.

\begin{tabular}{|c|c|c|c|c|}
\hline Class time line & $1: 00 \mathrm{~m}$ & $6: 00 \mathrm{~m}$ & $11: 00 \mathrm{~m}$ & $16: 00 \mathrm{~m}$ \\
\hline $\begin{array}{l}\text { SG hands on } \\
\text { T visits SG }\end{array}$ & & & & 1 \\
\hline $\begin{array}{l}\mathrm{T} \text { or } \mathrm{S} \text { mentions } \\
\text { crucial concept }\end{array}$ & & & & \\
\hline $\begin{array}{l}\text { Response misc } \\
\text { conceptual dif }\end{array}$ & & & |l|II I & \\
\hline $\begin{array}{l}\text { T supports } \\
\text { key vis feature }\end{array}$ & & & | |||| || | & \\
\hline $\begin{array}{l}\text { S supports } \\
\text { key vis feature }\end{array}$ & $\|$ & & $\underset{\mid}{|I| I} \mid$ & \\
\hline
\end{tabular}

FIG 6. Codes clustered around teacher visit to small group.

These results suggest a hypothesis that could help explain the pre-post results: that there are complementary strengths and weaknesses in the two lesson formats. We believe that more research is needed to determine when and for whom each format might best be used. In addition to hands-on-controls experience with animations, we suggest that students, especially those who may need support for recognizing and interpreting the meaning of visual features, might benefit from at least some whole class discussion with the computer visuals. We observed teachers using a number of teaching strategies to accomplish this and have included those strategies on our website [10].

\section{ACKNOWLEDGEMENTS}

This material is based upon work supported by the National Science Foundation under Grants DRL-1222709 and DRL-0723709, John J. Clement, PI. Any opinions, findings, and conclusions or recommendations expressed in this paper are those of the author(s) and do not necessarily reflect the views of the National Science Foundation.

(2002).

[6] M. Cook, Sc. Educ. 90, 1073 (2006).

[7] S. Schwan and R. Riempp, Learn. Instr. 14, 293 (2004).

[8] J. Bennett, S. Hogarth, F. Lubben, B. Campbell, and A. Robinson, Int. J. Sci. Educ. 32, 69 (2010).

[9] A. L. Stephens, Ed.D. thesis, University of Massachusetts, 2012

[10] http://www.umass.edu/teachingstrategies/ 